\title{
Megaphages infect Prevotella and variants are widespread in gut microbiomes
}

\author{
Audra E. Devoto', Joanne M. Santini ${ }^{\circledR 2}{ }^{2}$, Matthew R. Olm³ ${ }^{3}$ Karthik Anantharaman ${ }^{1,12}$, Patrick Munk ${ }^{4}{ }^{4}$, \\ Jenny Tung5 ${ }^{5}$, Elizabeth A. Archie ${ }^{6}$, Peter J.Turnbaugh ${ }^{7,8}$, Kimberley D. Seed ${ }^{3,8}{ }^{\text {, Ran Blekhman }}{ }^{9}$, \\ Frank M. Aarestrup ${ }^{(1)}{ }^{4}$, Brian C. Thomas ${ }^{1}$ and Jillian F. Banfield 1 1,10,11*
}

\begin{abstract}
Bacteriophages (phages) dramatically shape microbial community composition, redistribute nutrients via host lysis and drive evolution through horizontal gene transfer. Despite their importance, much remains to be learned about phages in the human microbiome. We investigated the gut microbiomes of humans from Bangladesh and Tanzania, two African baboon social groups and Danish pigs; many of these microbiomes contain phages belonging to a clade with genomes $>540$ kilobases in length, the largest yet reported in the human microbiome and close to the maximum size ever reported for phages. We refer to these as Lak phages. CRISPR spacer targeting indicates that Lak phages infect bacteria of the genus Prevotella. We manually curated to completion 15 distinct Lak phage genomes recovered from metagenomes. The genomes display several interesting features, including use of an alternative genetic code, large intergenic regions that are highly expressed and up to 35 putative transfer RNAs, some of which contain enigmatic introns. Different individuals have distinct phage genotypes, and shifts in variant frequencies over consecutive sampling days reflect changes in the relative abundance of phage subpopulations. Recent homologous recombination has resulted in extensive genome admixture of nine baboon Lak phage populations. We infer that Lak phages are widespread in gut communities that contain the Prevotella species, and conclude that megaphages, with fascinating and underexplored biology, may be common but largely overlooked components of human and animal gut microbiomes.
\end{abstract}

$\mathrm{H}$ uman and animal microbiomes are of enormous interest, given that microbial activity impacts nutrition, physiological development and disease ${ }^{1}$. The human gut microbiome has been intensively studied, mostly using gene fingerprinting methods to resolve body site specificity and microbiome compositional changes with age, health conditions and $\operatorname{diet}^{2-4}$. Less commonly applied is genome-resolved metagenomics, which involves simultaneous recovery of draft, and sometimes complete, genomes from metagenomes. Such studies have provided access to bacteriophage (phage), virus and plasmid sequences that are not accessible via fingerprinting methods ${ }^{5-7}$.

Phages are increasingly recognized as ubiquitous components of microbiomes. They can dramatically shape ecosystem structure via strain-specific predation, mediate horizontal gene transfer and redistribute nutrients by lysing host cells ${ }^{8}$. We investigated microbial communities in the gastrointestinal tracts of ten arsenic-impacted men from Laksam Upazila, Bangladesh to identify gut microbiomeassociated phages, link them to bacterial hosts and evaluate their distribution. We discovered phages with genomes that are exceptionally large, $>540$ kilobase pairs $(\mathrm{kb})$ in length (referred to as 'megaphages' in this study). As of June 2016, only 93 phages with genomes $>200 \mathrm{~kb}$ ('jumbo phages') were isolated, and none have a genome $>500 \mathrm{~kb}^{9}$. The average length of complete phage genomes is $53,644 \pm 45,677 \mathrm{bp}$, consistent with the average length of isolated double-stranded DNA viruses $(44,296 \pm 83,777 \mathrm{bp})^{10}$. We refer to the megaphages discovered in the Laksam Upazila cohort as Lak phages and determined that they replicate in Prevotella species, bacteria that tend to be enriched in the gut microbiomes of individuals who consume non-Western diets ${ }^{11}$. To determine whether these phages are common in other human and animal microbiomes, we investigated several DNA read data sets from samples containing abundant Prevotella. Overall, our results indicate that Lak phages are common and probably important components of the gut microbiomes of humans and animals.

\section{Results}

Megaphages identified in the gut microbiomes of Bangladeshi adults. We sequenced DNA from the faecal samples of ten adults living in Eruani village, Laksam Upazila, Bangladesh (Supplementary Table 1). Taxonomic classification and relative abundance information reveal that the communities are mostly dominated by Prevotella species (Supplementary Fig. 1). From individuals 20 and 22 we identified large genome fragments that were identified as phages (Supplementary Information) and selected for manual assembly curation (Methods). Two bioinformatically verified, circularized phage genomes, A1 and A2, were $>541 \mathrm{~kb}$ in length, close to the maximum size ever reported for a phage ${ }^{10}$. There was no evidence for integration of these sequences into bacterial genomes (Supplementary Information). Given their extraordinary size and to distinguish them from jumbo phages $\left(>200 \mathrm{~kb}_{\text {genomes }}{ }^{9}\right)$, we

'Department of Earth and Planetary Science, University of California, Berkeley, Berkeley, CA, USA. ${ }^{2}$ Institute of Structural \& Molecular Biology, Division of Biosciences, University College London, London, UK. ${ }^{3}$ Department of Plant and Microbial Biology, University of California, Berkeley, Berkeley, CA, USA. ${ }^{4}$ National Food Institute, Technical University of Denmark, Lyngby, Kongens, Denmark. ${ }^{5}$ Department of Evolutionary Anthropology, Duke University, Durham, NC, USA. ' Department of Biological Sciences, University of Notre Dame, Notre Dame, IN, USA. 'Department of Microbiology and Immunology, University of California, San Francisco, San Francisco, CA, USA. ${ }^{8}$ Chan Zuckerberg Biohub, San Francisco, CA, USA. ' Department of Genetics, Cell Biology, and Development, University of Minnesota, Minneapolis, MN, USA. ${ }^{10}$ The University of Melbourne, Melbourne, Victoria, Australia. ${ }^{11}$ Innovative Genomics Institute, University of California, Berkeley, Berkeley, CA, USA. 12Present address: Department of Bacteriology, University of Wisconsin-Madison, Madison, WI, USA. *e-mail: jbanfield@berkeley.edu 


\begin{tabular}{|c|c|c|c|c|c|c|}
\hline Phage & Sample of origin & Guanine-cytosine, \% & Length (bp) & No. tRNAs & $\begin{array}{l}\text { No. tRNA } \\
\text { introns }\end{array}$ & $\begin{array}{l}\text { No. predicted open reading } \\
\text { frames (code 15) }\end{array}$ \\
\hline A1-i & As cohort 22, no. 2 & 25.9 & 541,643 & 33 & 3 & 581 \\
\hline A1-ii & As cohort 22, no. 3 & 25.9 & 541,664 & 33 & 3 & 581 \\
\hline A1-iii & As cohort 22, no. 4 & 25.9 & 541,664 & 33 & 3 & 584 \\
\hline A1-iv & As cohort 22, no. 5 & 25.9 & 541,664 & 33 & 3 & 581 \\
\hline $\mathrm{A} 2$ & As cohort 20, no. 3 & 26.0 & 541,299 & 34 & 4 & 581 \\
\hline C1 & Cholera CH_A02_001D1 & 25.8 & 540,217 & 32 & $2^{\mathrm{a}}$ & 591 \\
\hline B1 & Baboon F22 (V) & 26.0 & 547,991 & 30 & 1 & 591 \\
\hline B2 & Baboon F3 (V) & 26.0 & 549,839 & 31 & 1 & 594 \\
\hline B3 & Baboon M09 (V) & 26.0 & 546,746 & 30 & 1 & 590 \\
\hline B4 & Baboon F30 (V) & 26.0 & 550,552 & 31 & 1 & 594 \\
\hline B5 & Baboon F18 (V) & 26.7 & 543,529 & 31 & 1 & 583 \\
\hline B6 & Baboon F16 (V) & 25.8 & 546,689 & 30 & 1 & 588 \\
\hline B7 & Baboon F11 (M) & 26.0 & 550,702 & 31 & 1 & 599 \\
\hline B8 & Baboon F4 (V) & 26.0 & 551,627 & 31 & 1 & 600 \\
\hline B9 & Baboon F01 (V) & 26.0 & 550,053 & 30 & 1 & 593 \\
\hline
\end{tabular}

${ }^{a}$ Variants within incomplete genomes have tRNA introns not found in the C1 genome. Baboons are from two social groups, V (Viola's) and M (Mica's) (see Tungi et al. $\left.{ }^{16}\right)$.

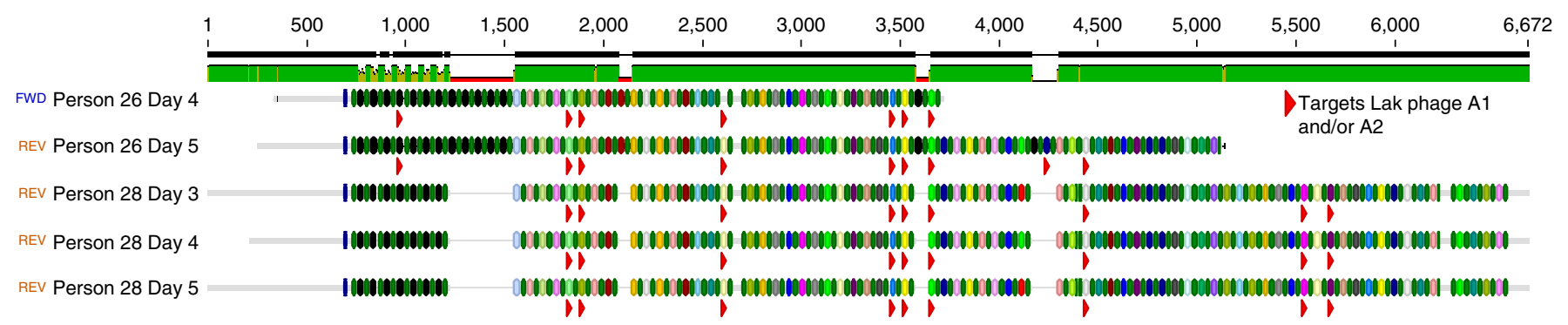

Fig. 1 | Alignment of the CRISPR arrays on four Prevotella scaffolds containing repeat GGTTTAATCGTACCTTTATGGAATTGAAAT. The green rods indicate repeats, the coloured rods indicate spacers. The same colour indicates the same spacer sequence, except for black rods, which indicate spacers different between individuals 26 and 28 (probably added to the diversifying locus ends). The red arrows indicate spacers targeting megaphages (also see Supplementary Fig. 3).

refer to these as 'megaphages'. The A1 and A2 genomes are largely syntenic and share $91.3 \%$ average nucleotide identity (ANI). They encode $\sim 35$ putative transfer RNA (tRNA) genes (Table 1), many of which are concentrated in specific genomic regions. Four nearidentical A1 phage genomes were independently curated to completion from samples taken on consecutive days from individual 22 (Table 1). The identified sequence variation occurred as population heterogeneity in all samples. Variant frequency analysis confirmed that the polymorphic sites are not linked; shifting relative abundances of subpopulations suggests ongoing replication over the four days (Supplementary Fig. 2).

Prevotella species are predicted as the hosts for megaphages based on CRISPR targeting. We used CRISPR targeting ${ }^{12}$ to identify Prevotella as the megaphage host (Fig. 1 and Supplementary Fig. 3, Supplementary Information). Given that many of the individuals have gut microbiomes dominated by Prevotella, we tested for megaphages in all the Laksam Upazila microbiome samples and found evidence for them in samples from individuals 21 and 23 (Supplementary Information). We attempted to isolate the megaphages using faecal material and Prevotella copri DSM $18205^{13}$ but isolation was unsuccessful (Supplementary Information).
Megaphages occur in other gut microbiomes. In a prior study, faecal samples were collected from a cohort of Bangladeshi cholera patients who were hospitalized in Dhaka, Bangladesh in 2016, but the reads were not assembled ${ }^{4}$. We conducted genome-resolved metagenomic analyses of these data sets. Many of the gut microbiomes were dominated by Prevotella and contained phages related to the A1 and A2 megaphages. One 540,217 kb genome, C1, was manually curated to completion. A data set from a second Bangladeshi cohort comprising six cholera-impacted adults was sampled from the same hospital in 2011. Of these, S75 had relatively abundant phages related to $\mathrm{C} 1$, and $\mathrm{S} 71$ and $\mathrm{S} 72 \mathrm{had}>100$ reads map to the $\mathrm{C} 1$ genome (Supplementary Fig. 4).

Faecal samples from individuals from the Hadza tribe of Tanzania were sequenced in a prior study ${ }^{14}$. Three of the 27 Hadza individuals had megaphages in sufficiently high abundance for genome assembly (Supplementary Fig. 4). Our assemblies were highly fragmented, but sequences shared $\sim 90 \%$ identity to phage A1 (Supplementary Table 1). Two samples from a previously sequenced cohort of Indian children ${ }^{15}$ also contained evidence of the megaphages (reads covered $>50 \mathrm{~kb}$ of the A1 genome).

Previously published metagenomic shotgun sequencing data sets from the faecal samples of 48 members of two social groups of Kenyan yellow baboons (Papio cynocephalus; one metagenome per 


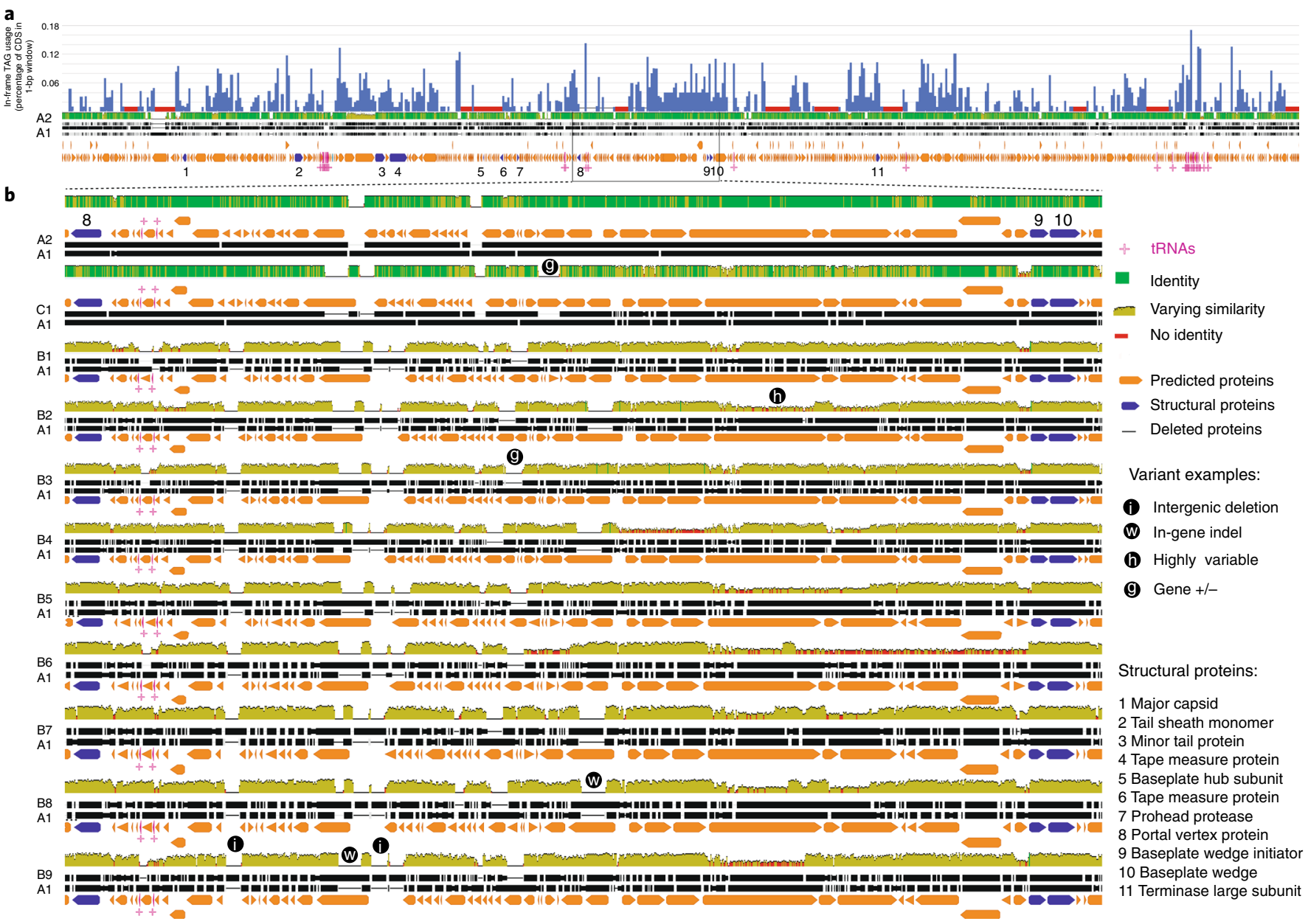

Fig. 2 Genomic features and variation in Lak phage genomes. a, Frequency of use of the TAG repurposed stop codon overlying the alignment of the A1 and $A 2$ genomes. The red bars indicate regions $>5 \mathrm{~kb}$ without TAG codon use. Structural proteins (dark blue) are mostly encoded in regions with high TAG use. CDS, coding sequence. The boxed region is shown in detail in $\mathbf{b}$. $\mathbf{b}$. Alignments of each distinct Lak phage genome against the A1 genome. A subset of this region was used in the pig Lak phage genome fragment alignments (Supplementary Fig. 6).

individual) were assembled and investigated to identify megaphage sequences ${ }^{16}$. Megaphages were detected in 43 of the 48 baboon gut microbiomes, and all samples contained multiple Prevotella strains or species (Supplementary Fig. 5). Sixteen high-quality genome bins were identified from 16 distinct samples, nine of which were curated to completion (B1-B9). All genomes were $>543 \mathrm{~kb}$ in length, and one (B8) is the largest phage genome reported in this study $(551,627 \mathrm{bp})$. All encode either 31 or 32 putative tRNAs (Table 1 , Supplementary Table 2 and Supplementary Information).

We analysed sequence data from Prevotella-containing samples from Danish pigs (Supplementary Table 1). Despite genome fragmentation, we identified a total of 18.7 mega base pairs $(\mathrm{Mb})$ of megaphage sequences with an alignment length of $15.9 \mathrm{Mb}$ to the A1 genome (one bin comprises a $462 \mathrm{~kb}$ sequence). At least $2 \mathrm{~kb}$ of an aligned megaphage sequence was detected in 104 of the 105 metagenomes. The pig-derived sequences span the A1 genome (Supplementary Fig. 6c). Thus, megaphages related to those present in humans and baboons also colonize pigs. Reads from 4 of $27 \mathrm{cow}$ rumen metagenomes ${ }^{17}$ mapped with low coverage across the entire A1 phage genome (Supplementary Table 1). However, analyses of 34 Prevotella-rich metagenomes from faecal samples from Tunapuco, a traditional agricultural community in the Andean highlands, did not detect megaphages ${ }^{18}$.

We identified a diversity of Prevotella strains via $16 \mathrm{~S}$ ribosomal RNA (rRNA) gene phylogenetic analysis. However, we found no clear link between cohort type and Prevotella species, or Prevotella species and megaphages (Supplementary Fig. 7).

Megaphage use an alternative genetic code. A notable feature of the megaphage genomes was their low coding density $(<70 \%$ for $\mathrm{A} 1$ and A2) when genes were predicted using the normal bacterial code (code 11). Fragmentation of many predicted proteins indicated that megaphages might be using an alternative genetic code. We determined that the canonical TAG stop codon is probably repurposed to encode glutamine, Q (code 15, Supplementary Fig. 8, see Methods), and confirmed this using the Fast and Accurate genetic Code Inference and Logo tool ${ }^{19}$. Code 15 was once previously reported for phages from metagenomes ${ }^{20}$. The TAG codon is not used in large parts of the A1 and A2 genomes, but it is used in some regions, including most that encode structural proteins (Fig. 2a and Supplementary Fig. 9). In samples from all days, we detected expression in regions encoding genes that do and do not use the TAG codon. Thus, if genes encoding structural proteins are expressed late, the phages in each sample are in a variety of stages of replication (Supplementary Fig. 9D).

Genomes with repurposed stop codons typically encode a suppressor tRNA. Multiple types of suppressor tRNAs were predicted (Supplementary Information and Supplementary Table 2), including one with a CTA anticodon that is necessary to repurpose the TAG stop codon. All complete megaphage genomes also encode 


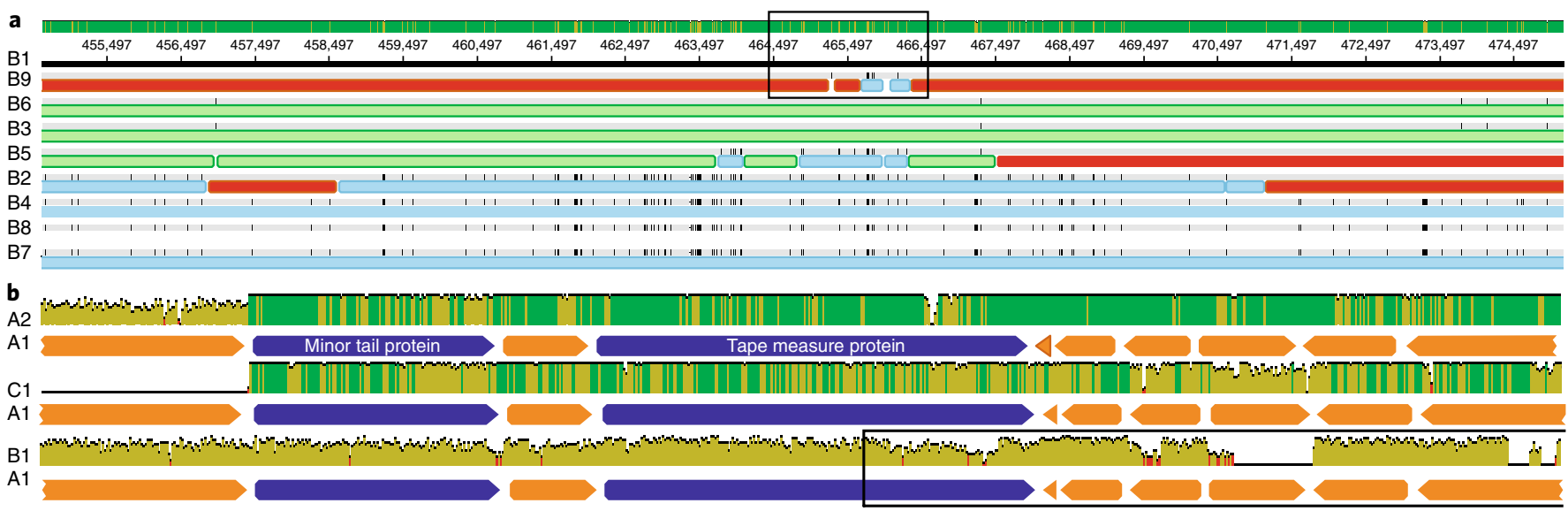

Fig. 3 | Comparison of B-Lak phage genomes reveals identical sequence blocks in a subset of the B-Lak phage. a, Sequence variation in a $20 \mathrm{~kb}$ region of the aligned B-Lak phage genomes with B1 as the reference. The coloured bars underline blocks with a shared sequence. Note evidence of admixture of sequence blocks, indicative of extensive homologous recombination among phages sampled from individual baboons. For the full alignment of the nine complete B-Lak phage genomes, see Supplementary Fig. 13. The box indicates the B9 region examined in detail (Supplementary Fig. 14). b, Relatively conserved and divergent regions in A1, A2 and C1. The open box indicates the region shown in $\mathbf{a}$.

release factor 2, which terminates translation by recognizing the TGA and TAA, but not TAG, stop codons. Thus, megaphages have the cellular machinery necessary to successfully translate genes with in-frame recoded TAG.

Comparative megaphage genomics. Terminase proteins are important during capsid assembly. Based on phylogenetic analyses, megaphage terminases place generally within the Myoviridae. Since they are clearly a divergent clade and highly distinct in terms of their consistently very large genomes and use of alternative coding, we define them as the 'Lak phages', named after Laksam Upazila, Bangladesh from where they were first detected.

The A1, A2 and $\mathrm{C} 1$ genomes are syntenic, as are all baboon Lak (B-Lak) genomes, but six large rearrangements distinguish the B-Lak from the A-Lak and C1-Lak genomes (Supplementary Fig. 10). As expected based on their synteny, A1 and A2 are more similar to $\mathrm{C} 1$ than the B-Lak genomes (Supplementary Table 3, Supplementary Fig. 11). Alignment of $\sim 70 \mathrm{~kb}$ region from each genome with the A1 genome (Fig. 2b) shows that insertions/deletions of sequence blocks within the central regions of genes (also see Supplementary Fig. 12), complete gene insertion/deletions, intergenic insertions/ deletions and varying levels of nucleotide substitutions (sometimes varying greatly within a gene, Supplementary Fig. 12) distinguish the genomes.

The nine B-Lak genomes share ANI values between 88.5 and $99.9 \%$ with one another (Supplementary Table 3). For comparison, A1 and A2 share $95 \%$ ANI (Supplementary Table 3). However, over an alignment with A1 (Fig. 2), B-Lak genomes share $61-65 \%$ ANI and $\mathrm{C} 1$ share $\sim 88 \%$ ANI. Notably, the majority of pig Lak genome fragments share $>90 \%$ sequence identity with A1, genome-wide (Supplementary Fig. 6).

Comparison of B-Lak genomes with one another revealed identical sequence blocks up to tens of kilobases in length in a subset of the B-Lak phage (Supplementary Fig. 13 and Fig. 3a). However, in adjacent regions, hundreds of single nucleotide polymorphisms (SNPs) distinguish these genotypes. These divergent sequences are often shared by a different subset of B-Lak genomes. The strong signal of sequence block admixture clearly indicates reassortment.

Lak phage populations are near-clonal, but some contain sequences of different Lak phages. We analysed sequence variation in reads mapped to each B-Lak genome (Supplementary Table 4) and found that $94-96.4 \%$ of the reads map to the genome with zero SNPs, providing confidence that the reported genomes are not chimeras of population variants. However, $0.01-0.8 \%$ of reads and read pairs in each data set match the sequences of other B-Lak genomes (Supplementary Table 4 and Supplementary Fig. 14). A subset of the reads (especially from B4, B7 and B9) probably derived from B-Lak genomes not reconstructed to date. In a few instances, adjacent SNP groups within individual Illumina reads directly indicate reassortment of alleles via homologous recombination (Supplementary Fig. 15).

The evidence of extensive homologous recombination motivated the question of whether phage relatedness (itself partially due to recombination) and phage admixture (sub-dominant genotypes within each population) could be predicted by baboon relatedness or social behaviour (Supplementary Table 5). Based on genetic (pedigree-based) estimates of kinship, grooming interactions (previously linked to similarities in baboon gut microbiomes, see Tungi et $\mathrm{al}^{16}{ }^{16}$ ) and host spatial proximity, there is no strong indication that these factors have strongly influenced the current phage genomes or within-population variation (Supplementary Fig. 16).

Lak phages encode tRNAs with highly conserved introns. Interestingly, Lak phage genomes have tRNAs with introns. To our knowledge, introns in phage tRNAs have not been reported previously. Several occur in A1, A2 and C1 tRNAs and one occurs in tRNA Tyr (GTA) in the baboon Lak phage genomes (Table 1 and Supplementary Table 6). The set of tRNA introns in the A1, A2 and $\mathrm{C} 1$ genomes only partially overlap. Intriguingly, however, where the same intron occurs in the same tRNA, its sequence is typically identical across cohorts (Supplementary Information).

We predicted the tRNA intron sequences and found that a putative tRNA Thr (TGT) is predicted to encode a possible tRNA (Supplementary Table 6), the sequence of which is preserved perfectly in all but one case across cohorts. $\mathrm{C} 1$ and other phage fragments from the cholera cohort lack this tRNA intron. Alignment of sequences with and without this intron reveals that the intron is offset by one nucleotide compared to the predicted intron (Fig. 4).

Lak phage metabolism and impact on host population dynamics. The vast majority of Lak protein coding genes are hypothetical (Supplementary Table 7). The largest inventory of genes with 


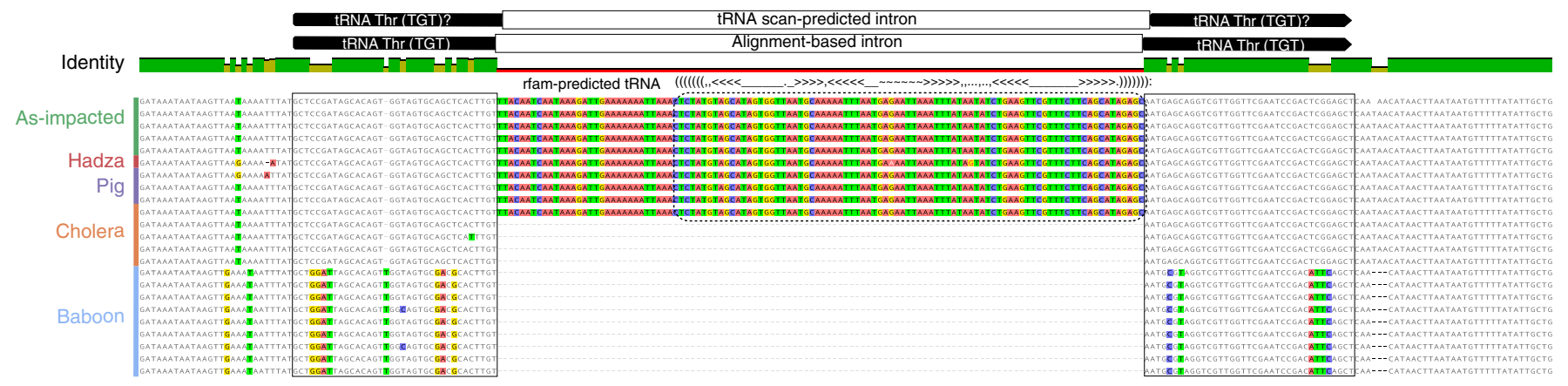

Fig. 4 | Alignment of sequences with a possible tRNA Thr (TGT). Some tRNAs are predicted to contain an intron that itself may encode a tRNA (dashed box with superimposed secondary structure). With the exception of the Hadza sequence, all introns are identical.

tentatively recognizable functions are involved in nucleotide, DNA and RNA transformations, functions previously noted as prominent in large phage genomes ${ }^{9}$. Phages may augment host translational machinery using sigma factors, translation initiation factors (for example, prokaryotic initiation factor-1), chain release factors as well as some genes that modify tRNAs (See Supplementary Information).

Given that a susceptible Prevotella host probably lacks CRISPRbased immunity, it is difficult to link Lak to the strain they are replicating in and thus to confidently infer the impact of phage predation on microbial community structure. Thus, we compared the abundances of all (rather than specific) Prevotella strains and Lak phage abundances over 4 time points for individuals 20 and 22. In both cases, phages are most abundant in the first sample and decline in abundance in the second sample, with corresponding increases in abundances of the more abundant Prevotella genotypes (Supplementary Fig. 17). Prevotella species probably differ to some extent in their metabolic capacities, growth rates and/ or nutrient preferences; changes in relative abundances of species as well as their cumulative abundance could alter overall gut microbiome function. Based on the limited observations, shifts in Prevotella abundances due to phage predation may occur on the day-to-day timescale.

\section{Discussion}

Lak phages are common yet overlooked members of gut microbiomes. Another widespread phage recently discovered using metagenomics ${ }^{21}$, crAssphage, infects Bacteroides, bacteria typically associated with a Western diet. Lak phages infect Prevotella species, which are often abundant in the gut microbiomes of animals and humans consuming a high-fibre, low-fat $\operatorname{diet}^{11}$. Notably, the 15 complete curated Lak phage genomes are $>5$ times larger than the crAssphage phage genome $e^{21}$ and only $\sim 4.6$ times smaller than the genomes of their Prevotella hosts (Supplementary Information).

Why have megaphages that infect Prevotella been overlooked, and why are megaphages of any kind so rarely described? As suggested previously, very large phages are difficult to isolate due to restricted mobility on plates used for plaque assays ${ }^{22}$. Genome fragmentation hinders their detection via metagenomics. Further, phage structural proteins may be obscured by distant homology to known sequences and gene fragmentation when predicted using the wrong genetic code. Based on a meta-analysis of public data, Paez-Espino et al. ${ }^{10}$ produced a data set that included fragments classified as very large phage genomes. However, 11 of the sequences $>200 \mathrm{~kb}$ are artefactual composites of identical repeated sequences (Supplementary Fig. 18). This underscores the importance of curation. Despite this, we suspect that many more phages with very large genomes will be uncovered in future metagenomic analyses.
Environmental distribution and dispersal of Lak phages. Have Lak phages co-evolved with their hosts or has there been facile dispersal across animal habitat types? The Lak phages found in humans and baboons are less closely related than those found in humans and pigs (Supplementary Fig. 11). We did not detect patterns of Prevotella speciation consistent with animal host specificity (Supplementary Fig. 7), so we suspect that Lak phages as well as their bacterial hosts may be actively dispersing across animal habitats.

It was possible to probe the importance of homologous recombination in Lak phage evolution because multiple genomes were reconstructed from different baboons. The data clearly indicate extensive allele reassortment involving all of the analysed baboon phage populations. Presumably, recombination events require coinfection, that is, the coexistence of these huge genomes inside a Prevotella cell. We infer that recombination events are recent, based on the low frequencies of SNPs that distinguish otherwise identical sequence blocks in different B-Lak genomes, and we suspect it is ongoing, given the presence of minor recombinant variants within some populations. Overall, the results suggests that distinct phages were brought into contact relatively recently, possibly following migration from another animal reservoir. A similar phenomenon was previously reported in bacterial genotypes ${ }^{23}$. Consistent with the recent introduction of Lak phages is their prevalence in the baboon population and associated low level of CRISPR-based immunity.

If Prevotella and their megaphages migrate among animal and human microbiomes, they could carry with them genes that are relevant to human and animal health and the spread of disease. The concept of zoonotic viruses is well established, but there may be analogous phenomena involving phages. Phages can disseminate virulence factors between bacterial strains, including toxin-encoding genes responsible for many important diseases such as diphtheria, cholera, dysentery, botulism, food poisoning, staphylococcal scalded skin syndrome, necrotizing pneumonia or scarlet fever ${ }^{8,24}$ and propagate other genes of medical interest among animal reservoirs, such as those involved in antimicrobial resistance. The finding of related Lak phages in baboon, pig, cow and human populations suggests this possibility; the probability that it may occur is clearly increased where phages have huge genomes.

Possible drivers of megaphage evolution. Interestingly, Lak phage genomes are in the size range of those of many putative bacterial and archaeal symbionts (for example, candidate phyla radiation bacteria and DPANN archaea (Diapherotrites, Parvarchaeota, Aenigmarchaeota, Nanoarchaeota, Nanohaloarchaea $\left.{ }^{25}\right)$ ). Moreover, the nucleic acid-related functions predicted for Lak phage genes are similar to those predicted for genes of candidate phyla radiation bacteria ${ }^{25}$. Are jumbo phages and megaphages the consequence of random local genome expansion events, or might there 
be stabilizing forces that converge on a specific genome length? Because we generated complete genomes for phages from multiple distinct cohorts, we could document consistent genome sizes of $\sim 540-552 \mathrm{~kb}$, suggesting that evolutionary forces preserve large genome size. Particle size affects flocculation and attachment, and larger particles may be better retained in specific pore spaces in the gut environment compared to smaller particles. Clearly not all gut-associated phages are large, so at best, physical size can provide only a partial explanation.

The existence of megaphages motivates the general question of the costs and benefits to the phages of large genomes and the feedbacks that drive their evolution. Lak phage genomes encode many tRNAs, which could improve their replication success (see Supplementary Information), but the span of genome-encoding tRNAs is small. More probably, the hundreds of hypothetical proteins in the genomes may ensure successful phage replication in the face of host defence mechanisms and could also be important for increasing the host range.

Evolution of large phage genomes, and thus few expensive particles per replication cycle, could be an ecological strategy analogous to K- versus r-selection. Phages would normally be viewed as r-strategists, leveraging the advantage of many offspring to ensure high probability that a particle will find a host where it can replicate before loss of viability. For large phages, the countering trade-off of a shift towards K-selection could be improved survival as the result of the large capsid size. Potentially, this is because of the increased stability of larger capsids, for example, due to their smaller radius of curvature. Clearly, many factors could come into play, and direct experiments involving isolated phages and their hosts are required to understand the intriguing phenomenon of megaphages in human and other animal gut microbiomes.

\section{Conclusion}

Megaphages are overlooked members of human and animal gut microbiomes. Their existence substantially increases the representation of phages whose genetic repertoires blur the boundaries that separate bacteria, bacterial symbionts and parasites/mobile elements. Their genomes hint at a fascinating biology and as yet unexplored complexity in the dynamics of gut microbiomes.

\section{Methods}

Samples, DNA and RNA extractions, sequencing and read archive analysis. Faecal samples were obtained from 10 Bangladeshi men (aged between 27 and 52 years) living in the Eruani village, Laksam Upazila, Bangladesh (samples were taken in April 2016). Informed consent was obtained from all individuals. All individuals displayed signs of arsenicosis and were consuming arseniccontaminated drinking water. Samples were collected on 4 consecutive days (labelled days $2-5$ ) and stored at $-20^{\circ} \mathrm{C}$ until they were shipped to the Santini $\mathrm{Lab}$ at University College London on dry ice. Samples were stored at $-80^{\circ} \mathrm{C}$ until nucleic acid extractions were performed. DNA was isolated with the PowerFecal DNA Isolation Kit (MO BIO Laboratories) according to the manufacturer's instructions and stored at $-20^{\circ} \mathrm{C}$. DNA samples were sent to RTLGenomics on dry ice and prepared for sequencing using the KAPA HyperPlus Kit (KAPA Biosystems) following the manufacturer's protocol, except that the DNA was fragmented physically using a Bioruptor (Diagenode Diagnostics), instead of enzymatically. The resulting individual libraries were run on a Fragment Analyze (Agilent) to assess the size distributions of the libraries, quantified using a Qubit 2.0 Fluorometer (Thermo Fisher Scientific), and also quantified using the KAPA Library Quantification Kit (KAPA Biosystems). Individual libraries were then pooled equimolar into their respective lanes and loaded onto an HiSeq 2500 (Illumina) $2 \times 125 \mathrm{bp}$ flow cell and sequenced. RNA was extracted from all 4 samples from individuals 20 and 22 with the PowerMicrobiome RNA Isolation Kit (MO BIO Laboratories) according to the manufacturer's instructions and stored at $-80^{\circ} \mathrm{C}$ until they were shipped to the QB3 Center at the University of California Berkeley on dry ice for sequencing.

The cohort of cholera patients comprised 42 men, 3 women, and 2 male and 2 female children. These samples were previously sequenced; see Methods from David et al. ${ }^{4}$ for information regarding informed consent and sampling protocols. The original study was approved by the Ethical and Research Review Committees of the International Centre for Diarrhoeal Disease Research, Bangladesh and the Institutional Review Board of Massachusetts General Hospital.
The analysis of megaphages in pigs targeted samples in which faecal DNA from multiple pigs (Sus domesticus) on Danish pig farms were pooled before sequencing ( $n=105$ farms). Farm selection and sampling protocols were approved by the EFFORT consortium (Ecology from Farm to Fork Of microbial drug Resistance and Transmission). For details on study design (including randomization and blinding), see the Methods available from Munk et al. ${ }^{26}$.

The baboon cohort comprised 17 male adults and 31 female adults from two social groups. The baboons ( . cynocephalus) were a part of a long-term study tracking individual baboons from several social groups in the Amboseli ecosystem since 1971. Study design was approved by the Institutional Animal Care and Use Committee at Duke University (protocol no. A028-12-02) and Notre Dame (protocol no. 16-09-3339). For details regarding the Amboseli project, and the methods used for sample collection and processing for the baboon cohort, see the previously published study by Tung et al. ${ }^{16}$.

\section{Binning of draft genomes, genome curation and annotation. Bins were} constructed from scaffolds of $>1 \mathrm{~kb}$ in length based on the combination of genome guanine-cytosine content, coverage and a phylogenetic profile as described in Anantharaman et al. ${ }^{27}$. The phylogenetic profile was established based on geneby-gene comparison to a reference genome data $\operatorname{set}^{28}$ We identified all sequences that encoded ribosomal protein S3, a gene that occurs in a relatively conserved block of genes that encode ribosomal proteins, and used these sequences to profile the overall community composition (taxonomy and abundance). Putative phage scaffolds were identified based on the high fraction of proteins with no related sequence in the database or similarity to phage proteins, as well as the presence of genes encoding structural proteins. Very large genome fragments were selected for curation. In cases where these were substantially shorter than the final genome length, candidate fragment collections identified based on consistency of guaninecytosine content, coverage and phylogenetic profile were subjected to curation. Coverage values were determined by read mapping using Bowtie $2^{29}$ with default parameters for paired reads.

The first genome curation step involved identification of local assembly errors and either correction of the errors or gap insertion using ra2.py ${ }^{30}$. Curation of each genome was conducted independently and involved correction of local scaffolding errors and gaps, contig extension to enable joins and circularization, with manual resolution of regions of confusion. Reads from the sample were mapped to the scaffold assembled from that sample and unplaced paired reads used to extend ends and fill gaps. Curation was conducted in Geneious ${ }^{31}$. Regions of confusion were identified based on much longer than expected placement of paired reads or backwards mapping of paired reads. Reads were manually relocated and reoriented. The final curated sequences were visualized throughout to confirm complete and accurate coverage of each genome. Final genomes were checked to confirm the absence of large repeated sequences that could have confounded the assembly. The start position was chosen in a random region so as not to interrupt a gene. Later reconstructed genomes were adjusted so that the start positions corresponded to those of earlier assembled genomes.

Genes were predicted on scaffolds $>1 \mathrm{~kb}$ using Prodigal ${ }^{32}$, initially using genetic code 11. Subsequently, Lak phage genes were re-predicted using code 15. Initial functional predictions were established based on similarity searches conducted using the basic local alignment search tool (BLAST) against the UniProt Knowledgebase and UniRef100 database ${ }^{33}$, and the Kyoto Encyclopedia of Genes and Genomes (KEGG) ${ }^{34}$ and uploaded to ggKbase (https://ggkbase. berkeley.edu/project_groups/megaphage). In addition, genes were annotated by scanning via hmmsearch ${ }^{35}$ with a collection of KEGG Hidden Markov Models representative of KEGG orthologous groups. The majority of phage structural genes were not identified in our initial functional predictions. Thus, we searched for proteins with unknown functions against the National Center for Biotechnology Information (NCBI) non-redundant protein database using position-specific iterative BLAST to identify remote homologues. These sequences were then aligned and searched against the Pfam_A_v31.0 and TIGRFAMs_v15.0 databases using HHpred to assign functions ${ }^{36}$. The amino acid sequences of terminases were so divergent from the terminases of previously analysed phages that they could not be identified using standard functional prediction methods. We found the terminase gene by searching all predicted phage proteins against a protein database of terminase large subunits, taken from phages spanning several different families and including all identified terminase large subunit proteins from phages with genomes $>200 \mathrm{~kb}$ in length.

The tRNA genes were predicted using tRNA scan with bacterial settings ${ }^{37}$. tRNAs that were larger than expected and could not be assigned a classification were evaluated in terms of potential introns. Genes were re-predicted using the eukaryote settings (bacterial tRNA genes typically do not encode introns, so the program does not recognize them) to identify the tRNA type, anticodon and intron sequence. Intron excision points were re-evaluated based on the alignments of genes with and without inserted sequences. Intron sequences were tested for possible classification using $\mathrm{rfam}^{38}$.

Codon usage was calculated in consecutive $1 \mathrm{~kb}$ windows, and was reported as the percentage of a specific codon out of all codons in the coding region of that window. Calculations were done using a custom script, cu.py. 
CRISPR targeting analyses. CRISPR arrays were predicted on all scaffolds $>1 \mathrm{~kb}$ in the Laksam Upazila cohort and the baboon cohort using a command line version of the program CRISPRDetect ${ }^{39}$ with parameter -array_quality_score cutoff $=3$. Only arrays with a score above the cut-off of 3 were considered. Spacers and repeat regions were extracted from the output files, and all spacers and repeats were searched against the Lak phage genomes A1 and A2 using BLASTn with the parameter - task $=$ short. No repeat regions had a hit to A1 or A2, so all spacers with a hit containing $\leq 1$ mismatches and a length $>24$ bp were considered to target Lak phages. The taxonomy of the scaffolds containing the CRISPR arrays with spacers targeting a Lak phage genome were determined by assigning taxonomy to all genes on the scaffold based on USEARCH clustering with the UniProt database. Scaffold taxonomy was assigned according to the highest taxonomic level shared by at least $50 \%$ of the genes on the scaffold. CRISPR arrays containing the repeat GGTTTAATCGTACCTTTATGGAATTGAAAT were chosen for reconstruction based on their high number of spacers targeting Lak phages. Arrays were manually aligned and spacers coloured using Geneious ${ }^{31}$.

Testing for megaphages in other data sets. Bacteria of the genus Prevotella are abundant in the gut microbiomes of humans in the developing world. Thus, we wondered if related megaphages occur in other gut microbiomes that contain Prevotella. A search of NCBI's non-redundant protein database for proteins related to those of the megaphages yielded no significant hits, so we selected individual metagenomic data sets from Prevotella-enriched samples for deeper analysis. Read data sets from previously published studies were selected based on the sampled environment and information about Prevotella content and downloaded from the NCBI's sequence read archive. Reads were mapped to the Lak phage genomes initially assembled from the Laksam Upazila cohort to determine whether or not Lak phages were present in the sample. Selected read sets were trimmed using sickle with default parameters (https://github.com/najoshi/sickle) and each data set was assembled separately using IDBA-UD ${ }^{30}$ with default parameters.

Phylogenetic and community compositional analyses. Community composition (Supplementary Fig. 1 and Supplementary Fig. 5) was determined by read mapping to the conserved ribosomal protein S3 gene (RPS3). The RPS3 genes were identified on all scaffolds $>1 \mathrm{~kb}$ in the Laksam Upazila Bangladeshi cohort and the baboon cohort, and classified to the species level based on USEARCH clustering ${ }^{40}$ with annotated proteins in the UniProt database. All RPS3 genes were then clustered at $90 \%$ identity using USEARCH, and a representative sequence from each cluster was chosen. Reads from each sample (all 10 people, 3 or 4 samples from consecutive days per person for the Bangladesh cohort and all 48 baboons) were mapped to these representative sequences, and the percentage coverage of each RPS3 gene was determined. Percentage coverage was then normalized by the sequencing depth of each sample to determine percentage project. Any genus that was present in $<10 \%$ cumulative abundance across all samples was grouped into the 'other' category. The stacked bar charts in Supplementary Figs. 1 and 5 were generated by plotting the percentage project of each genotypic variant in the same order for each sample, sorted by assigned genus. The bars were then coloured by genus, resulting in coloured genus bars divided by the genotypic variants within that genus (grey lines). The figure was plotted using the Matplotlib Python library ${ }^{41}$.

The Prevotella phylogenetic tree was constructed using 16S rRNA gene sequences. First, the Greengenes database ${ }^{42}$ of complete $16 \mathrm{~S}$ rRNA gene sequences was augmented with all $16 \mathrm{~S}$ rRNA gene sequences from Prevotella reference sequences on the NCBI that were independently classified as Prevotella (15 were assigned to a genus other than Prevotella and discarded). This augmented database was then used to classify $16 \mathrm{~S}$ rRNA gene sequences from all samples in each study where a megaphage was found, including samples in publicly available studies, using the assign_taxonomy.py script from qiimel and default parameters ${ }^{43}$. Sequences classified as Prevotella were aligned with all known reference Prevotella $16 \mathrm{~S}$ rRNA gene sequences and an Escherichia coli 16S rRNA gene outgroup (NCBI ref. J01859.1) using MUSCLE ${ }^{44}$. A tree was generated using RAxML-HPC2 on $\mathrm{XSEDE}^{45}$ on the CIPRES Science Gateway ${ }^{46}$ using parameters raxmlHPCHYBRID - T 4 -n result -s infile.txt -m GTRGAMMA -p 12345 -k -f a -N 100 -x 12345 --asc-corr lewis. The tree was edited and annotated with iTOL ${ }^{47}$.

Comparative genomics. Genome sequences were aligned using the progressiveMauve algorithm using default parameters ${ }^{48}$. In certain regions, the sequences were offset because the algorithm failed to align them. In some cases this could be corrected based on visual inspection. In other cases, the sequences were superimposed to constrain the overall alignment length; very low similarity scores were then displayed.

Predicted proteins from all known Lak phage genomes or bins were clustered along with predicted proteins from jumbo Sphingomonas phage PAU (ref: NC_019521.1) using MMseqs ${ }^{49}$. Fifteen protein subfamilies were identified that contained at least one protein from all Lak phage genomes or bins used. The terminase large subunit protein from phage PAU was clustered into a different subfamily from the Lak terminase large subunit proteins, and those two subfamilies were combined to generate 16 protein subfamilies used in further analysis. All subfamilies were manually curated to ensure only one protein from each Lak phage genome or bin or PAU was included. (In all cases where more than one protein from the same Lak phage genome or bin were grouped into the same subfamily, only the protein with the highest percentage identity to the rest of the subfamily was retained.) Proteins within each family were aligned using MUSCLE; alignments were concatenated according to the order the genes appeared in the A1 genome using Geneious ${ }^{31}$. A tree was generated using RAxML-HPC2 on $\mathrm{XSEDE}^{45}$ on the CIPRES Science Gateway ${ }^{46}$ using parameters raxmlHPC-HYBRID -T 4 -n result -s infile.txt - 12345 -m PROTGAMMADAYHOFF -f a -N 100 -x 12345 --asc-corr lewis. The tree was rooted using the concatenation derived from Sphingomonas phage PAU. The tree was edited and annotated with iTol ${ }^{47}$.

Pig genome fragment analysis. To search the 105 metagenomes constructed from collections of faecal samples from Danish pig farms, we aligned all the assembled pig metagenomic scaffolds $>1 \mathrm{~kb}$ in length against the Lak phage A1 reference genome using NUCmer (Mummer version 4.0.0beta). Filtering was done, so that alignments of at least $2 \mathrm{~kb}$ and $70 \%$ nucleotide identity were kept. The lengths of scaffolds meeting these criteria were summed to estimate the total genome sequence attributable to Lak phages, and the total alignment length was calculated. A metagenome was considered to contain Lak phages as long as at least one scaffold with an alignment length $>2 \mathrm{~kb}$ was identified.

Statement of ethics. The human faecal samples obtained were part of a clinical phase I/II study in rural Bangladesh entitled 'Selenium and arsenic pharmacodynamics' (SEASP) run by Graham George (University of Saskatchewan) and funded by the Canadian Federal Government, through a programme entitled Grand Challenges Canada-Stars in Global Health, with additional funds from the Global Institute for Water Security at the University of Saskatchewan. The SEASP trial was approved by the University of Saskatchewan Research Ethics Board (14-284) and the Bangladesh Medical Research Council (940,BMRC/NREC/20102013/291). Additional ethics approval was also obtained by UCL (7591/001).

Reporting Summary. Further information on research design is available in the Nature Research Reporting Summary linked to this article.

\section{Code availability}

The cu.py script is available at https://github.com/oddaud/cu.py.

\section{Data availability}

The 15 Lak phage genomes have been deposited at NCBI under BioProject PRJNA491720. The genomes can also be downloaded from https://ggkbase. berkeley.edu/project_groups/megaphage. The read archive and other accession information is provided in Supplementary Table 1. Please note that it is necessary to register for a ggKbase account by providing an email address before accessing or downloading the data.

Received: 28 June 2018; Accepted: 5 December 2018; Published online: 28 January 2019

\section{References}

1. Sommer, F. \& Bäckhed, F. The gut microbiota: masters of host development and physiology. Nat. Rev. Microbiol. 11, 227-238 (2013).

2. David, L. A. et al. Diet rapidly and reproducibly alters the human gut microbiome. Nature 505, 559-563 (2014).

3. Bäckhed, F. et al. Dynamics and stabilization of the human gut microbiome during the first year of life. Cell Host Microbe 17, 690-703 (2015).

4. David, L. A. et al. Gut microbial succession follows acute secretory diarrhea in humans. MBio 6, e00381-15 (2015)

5. Waller, A. S. et al. Classification and quantification of bacteriophage taxa in human gut metagenomes. ISME J. 8, 1391-1402 (2014)

6. Yutin, N. et al. Discovery of an expansive bacteriophage family that includes the most abundant viruses from the human gut. Nat. Microbiol. 3, 38-46 (2018).

7. Minot, S. et al. The human gut virome: inter-individual variation and dynamic response to diet. Genome Res. 21, 1616-1625 (2011).

8. Penadés, J. R., Chen, J., Quiles-Puchalt, N., Carpena, N. \& Novick, R. P. Bacteriophage-mediated spread of bacterial virulence genes. Curr. Opin. Microbiol. 23, 171-178 (2015).

9. Yuan, Y. \& Gao, M. Jumbo bacteriophages: an overview. Front. Microbiol. 8 403 (2017).

10. Paez-Espino, D. et al. Uncovering Earth's virome. Nature 536, 425-430 (2016).

11. Gupta, V. K., Paul, S. \& Dutta, C. Geography, ethnicity or subsistence-specific variations in human microbiome composition and diversity. Front. Microbiol. 8, 1162 (2017)

12. Andersson, A. F. \& Banfield, J. F. Virus population dynamics and acquired virus resistance in natural microbial communities. Science 320, 1047-1050 (2008).

13. Hayashi, H., Shibata, K., Sakamoto, M., Tomita, S. \& Benno, Y. Prevotella copri sp. nov. and Prevotella stercorea sp. nov., isolated from human faeces. Int. J. Syst. Evol. Microbiol. 57, 941-946 (2007). 
14. Rampelli, S. et al. Metagenome sequencing of the Hadza hunter-gatherer gut microbiota. Curr. Biol. 25, 1682-1693 (2015).

15. Ghosh, T. S. et al. Gut microbiomes of Indian children of varying nutritional status. PLoS ONE 9, e95547 (2014)

16. Tung, J. et al. Social networks predict gut microbiome composition in wild baboons. eLife 4, e05224 (2015).

17. Thomas, M. et al. Metagenomic characterization of the effect of feed additives on the gut microbiome and antibiotic resistome of feedlot cattle. Sci. Rep. 7, 12257 (2017)

18. Obregon-Tito, A. J. et al. Subsistence strategies in traditional societies distinguish gut microbiomes. Nat. Commun. 6, 6505 (2015).

19. Dutilh, B. E. et al. FACIL: Fast and Accurate genetic Code Inference and Logo. Bioinformatics 27, 1929-1933 (2011).

20. Ivanova, N. N. et al. Stop codon reassignments in the wild. Science $\mathbf{3 4 4}$ 909-913 (2014).

21. Dutilh, B. E. et al. A highly abundant bacteriophage discovered in the unknown sequences of human faecal metagenomes. Nat. Commun. 5, 4498 (2014).

22. Serwer, P., Hayes, S. J., Thomas, J. A. \& Hardies, S. C. Propagating the missing bacteriophages: a large bacteriophage in a new class. Virol. J. 4 21 (2007).

23. Denef, V. J. \& Banfield, J. F. In situ evolutionary rate measurements show ecological success of recently emerged bacterial hybrids. Science 336, 462-466 (2012).

24. Brown-Jaque, M., Calero-Cáceres, W. \& Muniesa, M. Transfer of antibioticresistance genes via phage-related mobile elements. Plasmid 79, 1-7 (2015).

25. Castelle, C. J. \& Banfield, J. F. Major new microbial groups expand diversity and alter our understanding of the tree of life. Cell 172, 1181-1197 (2018).

26. Munk, P. et al. Abundance and diversity of the faecal resistome in slaughter pigs and broilers in nine European countries. Nat. Microbiol. 3, 898-908 (2018).

27. Anantharaman, K. et al. Thousands of microbial genomes shed light on interconnected biogeochemical processes in an aquifer system. Nat. Commun. 7, 13219 (2016).

28. Sieber, C. M. K. et al. Recovery of genomes from metagenomes via a dereplication, aggregation and scoring strategy. Nat. Microbiol. 3, 836-843 (2018).

29. Langmead, B. \& Salzberg, S. L. Fast gapped-read alignment with Bowtie 2. Nat. Methods 9, 357-359 (2012).

30. Brown, C. T. et al. Unusual biology across a group comprising more than 15\% of domain Bacteria. Nature 523, 208-211 (2015).

31. Kearse, M. et al. Geneious Basic: an integrated and extendable desktop software platform for the organization and analysis of sequence data. Bioinformatics 28, 1647-1649 (2012).

32. Hyatt, D. et al. Prodigal: prokaryotic gene recognition and translation initiation site identification. BMC Bioinformatics 11, 119 (2010).

33. Suzek, B. E., Huang, H., McGarvey, P., Mazumder, R. \& Wu, C. H. UniRef: comprehensive and non-redundant UniProt reference clusters. Bioinformatics 23, 1282-1288 (2007).

34. Ogata, H. et al. KEGG: Kyoto Encyclopedia of Genes and Genomes. Nucleic Acids Res. 27, 29-34 (1999).

35. Finn, R. D., Clements, J. \& Eddy, S. R. HMMER web server: interactive sequence similarity searching. Nucleic Acids Res. 39, W29-W37 (2011).

36. Zimmermann, L. et al. A completely reimplemented MPI Bioinformatics Toolkit with a new HHpred server at its core. J. Mol. Biol. 430, 2237-2243 (2018).

37. Lowe, T. M. \& Chan, P. P. tRNAscan-SE On-line: integrating search and context for analysis of transfer RNA genes. Nucleic Acids Res. 44, W54-W57 (2016).

38. Griffiths-Jones, S., Bateman, A., Marshall, M., Khanna, A. \& Eddy, S. R. Rfam: an RNA family database. Nucleic Acids Res. 31, 439-441 (2003).

39. Biswas, A., Staals, R. H. J., Morales, S. E., Fineran, P. C. \& Brown, C. M. CRISPRDetect: a flexible algorithm to define CRISPR arrays. BMC Genomics 17, 356 (2016)

40. Edgar, R. C. Search and clustering orders of magnitude faster than BLAST. Bioinformatics 26, 2460-2461 (2010).

41. Hunter, J. D. Matplotlib: a 2D graphics environment. Comput. Sci. Eng. 9, 90-95 (2007).

42. DeSantis, T. Z. et al. Greengenes, a chimera-checked 16S rRNA gene database and workbench compatible with ARB. Appl. Environ. Microbiol. 72, 5069-5072 (2006).

43. Caporaso, J. G. et al. QIIME allows analysis of high-throughput community sequencing data. Nat. Methods 7, 335-336 (2010).

44. Edgar, R. C. MUSCLE: multiple sequence alignment with high accuracy and high throughput. Nucleic Acids Res. 32, 1792-1797 (2004).

45. Stamatakis, A. RAxML version 8: a tool for phylogenetic analysis and post-analysis of large phylogenies. Bioinformatics 30, 1312-1313 (2014).
46. Miller M. A., Pfeiffer W. \& Schwartz T. The CIPRES Science Gateway: a community resource for phylogenetic analyses. In Proc. 2011 TeraGrid Conference: Extreme Digital Discovery, 41 (ACM Publications, Salt Lake City, 2011).

47. Letunic, I. \& Bork, P. Interactive tree of life (iTOL) v3: an online tool for the display and annotation of phylogenetic and other trees. Nucleic Acids Res. 44, W242-W245 (2016).

48. Darling, A. C. E., Mau, B., Blattner, F. R. \& Perna, N. T. Mauve: multiple alignment of conserved genomic sequence with rearrangements. Genome Res. 14, 1394-1403 (2004).

49. Hauser, M., Steinegger, M. \& Söding, J. MMseqs software suite for fast and deep clustering and searching of large protein sequence sets. Bioinformatics 32, 1323-1330 (2016)

\section{Acknowledgements}

We thank T. Lowe for helpful discussion related to tRNAs and introns and D. Paez-Espino and colleagues at the Joint Genome Institute for generating sequences in a meta-analysis related to IMG-VR that enabled identification of the baboon cohort as a potential source of Lak phage sequences. We thank S. Lei and K. Lane for ggKbase support, L. Barreiro for his contributions to the baboon study and Dr. R. Meheust for his assistance with the protein clustering analysis. Funding was provided by the National Institutes of Health (RAI092531A) and Sloan Foundation (G 2012-10-05) to J.F.B., Chan Zuckerberg Biohub funding to J.F.B., P.T. and K.S., and National Science Foundation grant no. IOS 1053461 to E.A.A. We acknowledge Rampelli et al. ${ }^{14}$ Ghosh et al. ${ }^{15}$, Thomas et al. ${ }^{14,1517}$, and Obregon-Tito et al. ${ }^{18}$ whose published research generated, respectively, the Hadza, Indian children, cow rumen and Peruvian gut read data sets used in this study. Faecal samples were collected from patients in the clinical phase I/II SEASP trial in Bangladesh that was jointly led by G. George and I. Pickering (University of Saskatchewan), with the assistance of the SEASP team (https://clinicaltrials.gov/ct2/show/NCT02377635), and funded by the Canadian Federal Government, through Grand Challenges Canada-Stars in Global Health and by the Global Institute for Water Security. We thank O. Ponomarenko and S. Shaha for organizing the transport of the faecal samples.

\section{Author contributions}

The initial study was designed by J.M.S. and J.F.B. and refocused by A.E.D., J.F.B. and J.M.S. J.M.S. isolated the nucleic acids from the Laksam Upazila Bangladesh cohort and provided the DNA sequencing. K.D.S. provided the DNA sequencing for the second cholera-impacted cohort. J.F.B. and A.E.D. curated the genomes. A.E.D. constructed the phylogenetic trees and analysed their codon use, with input from B.C.T. and K.A. A.E.D. and J.F.B. conducted the comparative genomic analyses, with input from M.R.O. A.E.D. and J.F.B. analysed the predicted protein sequences. J.M.S. attempted the Lak phage isolations. P.M. and F.M.A. provided the pig metagenomic data, which was analysed by P.M. and J.F.B. J.T., E.A. and R.B. generated the previously published baboon reads and provided input to the metadata analysis. P.J.T. generated the previously published cholera-impacted cohort read data set. J.F.B. and A.D. wrote the manuscript, with input from J.M.S., M.R.O. and P.M. All authors read and approved the manuscript.

\section{Competing interests}

J.F.B. and B.C.T. are founders of Metagenomi. A.E.D. is an employee of Metagenomi as of 1 September 2018.

\section{Additional information}

Supplementary information is available for this paper at https://doi.org/10.1038/ s41564-018-0338-9.

Reprints and permissions information is available at www.nature.com/reprints. Correspondence and requests for materials should be addressed to J.F.B.

Publisher's note: Springer Nature remains neutral with regard to jurisdictional claims in published maps and institutional affiliations.

(C) The Author(s) 2019

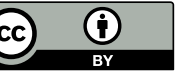

Open Access This article is licensed under a Creative Commons Attribution 4.0 International License, which permits use, sharing, adaptation, distribution and reproduction in any medium or format, as long as you give appropriate credit to the original author(s) and the source, provide a link to the Creative Commons license, and indicate if changes were made. The images or other third party material in this article are included in the article's Creative Commons license, unless indicated otherwise in a credit line to the material. If material is not included in the article's Creative Commons license and your intended use is not permitted by statutory regulation or exceeds the permitted use, you will need to obtain permission directly from the copyright holder. To view a copy of this license, visit http://creativecommons.org/licenses/by/4.0/. 


\title{
Reporting Summary
}

Nature Research wishes to improve the reproducibility of the work that we publish. This form provides structure for consistency and transparency in reporting. For further information on Nature Research policies, see Authors \& Referees and the Editorial Policy Checklist.

\section{Statistical parameters}

When statistical analyses are reported, confirm that the following items are present in the relevant location (e.g. figure legend, table legend, main text, or Methods section).
n/a Confirmed
$\square \bigotimes$ The exact sample size $(n)$ for each experimental group/condition, given as a discrete number and unit of measurement
$\square$ \ An indication of whether measurements were taken from distinct samples or whether the same sample was measured repeatedly
$\bigotimes$ The statistical test(s) used AND whether they are one- or two-sided
$\triangle \square$ Only common tests should be described solely by name; describe more complex techniques in the Methods section.
Х $\square$ A description of all covariates tested
Х $\square$ A description of any assumptions or corrections, such as tests of normality and adjustment for multiple comparisons
$\square \bigotimes \begin{aligned} & \text { A full description of the statistics including central tendency (e.g. means) or other basic estimates (e.g. regression coefficient) AND } \\ & \text { variation (e.g. standard deviation) or associated estimates of uncertainty (e.g. confidence intervals) }\end{aligned}$
$\bigotimes \square \begin{aligned} & \text { For null hypothesis testing, the test statistic (e.g. } F, t, r \text { ) with confidence intervals, effect sizes, degrees of freedom and } P \text { value noted } \\ & \text { Give } P \text { values as exact values whenever suitable. }\end{aligned}$
Х $\square$ For Bayesian analysis, information on the choice of priors and Markov chain Monte Carlo settings
Х $\square$ For hierarchical and complex designs, identification of the appropriate level for tests and full reporting of outcomes
$\bigotimes \square$ Estimates of effect sizes (e.g. Cohen's $d$, Pearson's $r$ ), indicating how they were calculated
$\varnothing$ Clearly defined error bars
\. $\square$ tate explicitly what error bars represent (e.g. SD, SE, CI)

Our web collection on statistics for biologists may be useful.

\section{Software and code}

\author{
Policy information about availability of computer code \\ Data collection Geneious 9.1.8 (Licensed, paid version used in this study, free versions available) \\ BBmap (Version: Last modified May 11, 2017) \\ IDBA_UD 1.1 .1 \\ Bowtie2 aligner 2.3.4.1
}

Data analysis

\author{
Prodigal V2.6.3 \\ usearch v10.0.240_i86linux64, 1057Gb RAM, 80 cores \\ tRNAscan-SE 2.0 \\ MUSCLE v3.8.31 \\ blastn: $2.6 .0+$ (command line version) \\ CRISPRDetect 2.2 (command line version) \\ Mummer 4.0.0beta \\ cu.py (available at https://github.com/oddaud/cu.py)
}

For manuscripts utilizing custom algorithms or software that are central to the research but not yet described in published literature, software must be made available to editors/reviewers upon request. We strongly encourage code deposition in a community repository (e.g. GitHub). See the Nature Research guidelines for submitting code \& software for further information. 
Policy information about availability of data

All manuscripts must include a data availability statement. This statement should provide the following information, where applicable:

- Accession codes, unique identifiers, or web links for publicly available datasets

- A list of figures that have associated raw data

- A description of any restrictions on data availability

The 15 Lak phage genomes have been deposited at NCBI under BioProject PRJNA491720. The genomes can also be downloaded from https://ggkbase.berkeley.edu/ project_groups/megaphage. Read archive and other accession information is provided in Table S1. Please note that it is necessary to register for a ggKbase account by providing an email address prior to accessing or downloading the data.

\section{Field-specific reporting}

Please select the best fit for your research. If you are not sure, read the appropriate sections before making your selection.

$\bigotimes$ Life sciences $\quad \square$ Behavioural \& social sciences $\square$ Ecological, evolutionary \& environmental sciences

For a reference copy of the document with all sections, see nature.com/authors/policies/ReportingSummary-flat.pdf

\section{Life sciences study design}

All studies must disclose on these points even when the disclosure is negative.

Sample size Pig cohort: $\mathrm{N}=105$ samples, where each sample is the pooled feces of multiple individual pigs

Laksam, Bangladesh cohort: $\mathrm{N}=10$ male adults, 3-4 samples per adult

Cholera-impacted cohort: $\mathrm{N}=42$ male adults, 3 female adults, 2 male and 2 female children

Baboon cohort: 17 male adults and 31 female adults

Data exclusions None

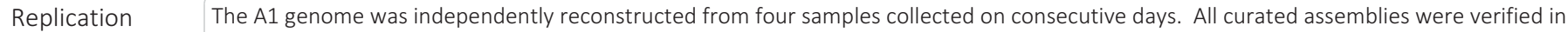
multiple read mapping steps.

Sample collection was not replicated except when multiple samples were collected from the same adult in the Laksam, Bangladesh cohort.

Samples from the same individual were more similar to each other than other individuals, as expected.

Randomization Randomization is not applicable because there were no experimental groups designated in this study.

Blinding Blinding was not performed because it was not applicable to this study. This study was a survey of various populations, and was not dependent on the presence / absence of certain characteristics.

\section{Reporting for specific materials, systems and methods}

Materials \& experimental systems

$\mathrm{n} / \mathrm{a}$ Involved in the study

\ $\square$ Unique biological materials

Х $\square$ Antibodies

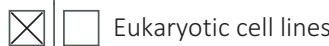

Х Palaeontology

Х $\square$ Animals and other organisms

$\square$ Х Human research participants

\begin{tabular}{l|l}
\multicolumn{2}{l}{ Methods } \\
\hline n/a Involved in the study \\
$\triangle$ \\
$\square$ ChIP-seq \\
$\square$ \\
$\square$ MRI-based neuroimaging
\end{tabular}

\section{Human research participants}

Policy information about studies involving human research participants

Population characteristics The only human subjects used in this study where from the Laksam, Bangladesh cohort. All other human and animal subjects were described by previous studies that had been published at the time of writing this paper. 
The Laksam, Bangladesh cohort consisted of 10 adult male subjects that were currently hospitalized. All subjects displayed signs of arsenicosis and were consuming arsenic-contaminated drinking water.

Recruitment

All patients were recruited do to their presentation of arsenicosis symptoms. Given that they were not healthy individuals and were in a diseases state, it is unknown how their disease contributed to the results of this study. However, the Lak phage reported here were found in numerous other environments including in livestock, so it is unlikely that the health of these individuals played a significant role in the presence/absence of Lak. Their health may have, however, played a role in the overall composition of their microbiome. 